\title{
Polygon Approximation Based Cognitive Information Delivery in Geo-location Database Oriented Spectrum Sharing
}

\author{
Zhiqing Wei, Huici Wu, Zhiyong Feng \\ Beijing University of Posts and Telecommunications \\ Beijing 100876, P. R. China \\ [e-mail: fengzy@bupt.edu.cn] \\ *Corresponding author: Zhiyong Feng
}

Received October 7, 2016; revised December 24, 2016; revised March 8, 2017; accepted April 2, 2017; published June 30, 2017

\begin{abstract}
In geo-location database oriented spectrum sharing system, the entire geographic area is divided into meshes to classify, store and delivery the cognitive information. In this paper, we propose a flexible polygon mesh division scheme to reduce the number of meshes. Hence the cognitive information can be reduced correspondingly. Besides, polygon mesh can approximate the real environment and reduce the error of cognitive information because the edges of polygon are selected along the boundaries of the networks. We have designed the polygon approximation algorithm and have analyzed the relation between the error and the number of polygon's edges. Finally, the simulation results are provided to verify the algorithm and analysis. The polygon mesh division scheme in this paper provides an efficient approach for cognitive information organization in database oriented spectrum sharing system.
\end{abstract}

Keywords: Spectrum sharing, database, polygon approximation.

This work is supported by the National Natural Science Foundation of China (No. 61601055, No. 61631003), and the National Science Fund for Distinguished Young Scholars (No. 61525101). 


\section{Introduction}

D riven by devices such as smart phones, tablet PC and the services such as social networking, streaming media and online game, the amount of mobile traffic is increasing exponentially. As the critical resource to improve the capacity of wireless networks, spectrum is always billion dollar asset. However, the licensed spectrum is not fully utilized [1], including the spectrum of TV broadcasting networks, which can be exploited by the unlicensed users. The standard IEEE 802.22 [2] was established to enhance the exploitation of TV white spaces when analog TV was replaced by digital TV. Hence there are various literatures studying spectrum sensing and spectrum sharing of TV white spaces under the standard IEEE 802.22 [3][4].

In order to discover TV white spaces, spectrum sensing and database based methods are proposed [5][6]. Paisana et al. in [7] argued that geo-location database based spectrum access technology is appropriate to exploit TV white spaces because the features of TV white spaces are relatively predictable. Both Federal Communications Commission (FCC) and European Conference of Postal and Telecommunications Administrations (CEPT) have released rules, orders and technical specifications to allow unlicensed radio transmitters or TV band devices (TVBDs) to operate at TV white spaces based on the geo-location database approach [8][9][10]. Upon the emergence of geo-location database, the construction of database and resource allocation scheme with assistance of database are addressed. Previously, we proposed a theoretical framework for the construction of cognitive database in [11] and [12]. Besides, Perez-Romero et al. in [13] adopted the radio environment map to management the interference in cognitive radio networks. Liu et al. in [14] designed the database based spectrum access and resource allocation scheme for cognitive radio networks.

However, as far as we know, although geo-location database in cognitive radio networks is widely studied, existing studies mainly focus on the construction and application of geo-location database, such as spectrum access and resource allocation. Efficient cognitive information delivery between database and unlicensed users is rarely studied. However, if this problem is not solved, the impact of database on the performance of cognitive radio networks is unknown. Caleffi et al. in [15] developped a stochastic analytical framework and designed optimal database access strategy, where the expected overall communication opportunities can be maximized. To deliver the cognitive information, some literatures designed the mesh based scheme [16][17][18]. When unlicensed user is within a mesh, it would query the database only once. However, when unlicensed user moves into a new mesh, it will query the database again for new cognitive information. Zhang et al. in [18] proposed a mesh division and differential information coding schemes to improve the efficiency of cognitive information delivery. However, the meshes are regular and cognitive information is not fully compressed. In [11][12], we proposed mesh fusion schemes that can further reduce the number of meshes and improve the efficiency of cognitive information delivery. [16][17][19] studied the impact of the size of meshes on the delivery of cognitive information. [20][21] suggested that the length of mesh's edge to be 30-200 meters. Overall, most literatures recommend the same size of $100 \mathrm{~m} \times 100 \mathrm{~m}$ for mesh division. However, we have verified that uniform mesh division is not flexible and there is still redundancy in congitive information. Therefore we designed the fractal based irregular mesh division scheme in [22]. Besides, it is noted that the literatures [23]-[25] also devoted to construct the geo-location database for cognitive radio networks. Braham et al. in [23] designed a scheme for coverage analysis of wireless networks, which is a 
critical step in the construction of geo-location database. Zou et al. in [24] developped a test bed named WinIPS for online radio map construction. Sodagari et al. in [25] studied the secure radio environment map, which aims to design the robust and secure database for dynamic spectrum access in cognitive radio networks.

In this paper, we propose a more flexible mesh division scheme, which adopts polygon mesh to classify cognitive information of entire area. It is noted that the number of meshes can be reduced significantly. Hence cognitive information can be reduced correspondingly. Besides, polygon meshes can well approximate the real environment and reduce the error of cognitive information compared with rectangle meshes because the edges of polygon are selected along the boundaries of networks in polygon approximation method. In this paper, we have designed polygon approximation algorithm. Besides, we have analyzed the relation between the error of cognitive information and the number of polygon's edges.

This paper is organized as follows. In Section 2, we describe the problem to be solved. In Section 3, the polygon approximation algorithm is introduced in details. The algorithm analysis is provoided in Section 4. The simulation and numerical results are provided in Section 5. Finally, in Section 6, we summarize this paper.

\section{Problem Statement}

\subsection{Cognitive Radio Database}

We consider a specific region where three TV networks exist. The TV network is primary network. Unlicensed user which is not covered by a TV network can use the spectrum of TV network. Therefore, in Fig. 1, the radio environment of different spots may be different. For example, in spot A, the spectrum band of network 3 is unavailable. However, the spectrum bands of network 1 and network 2 are available. In spot B, the spectrum bands of all TV networks are unavailable. But the spectrum bands of all TV networks are available in spot C.

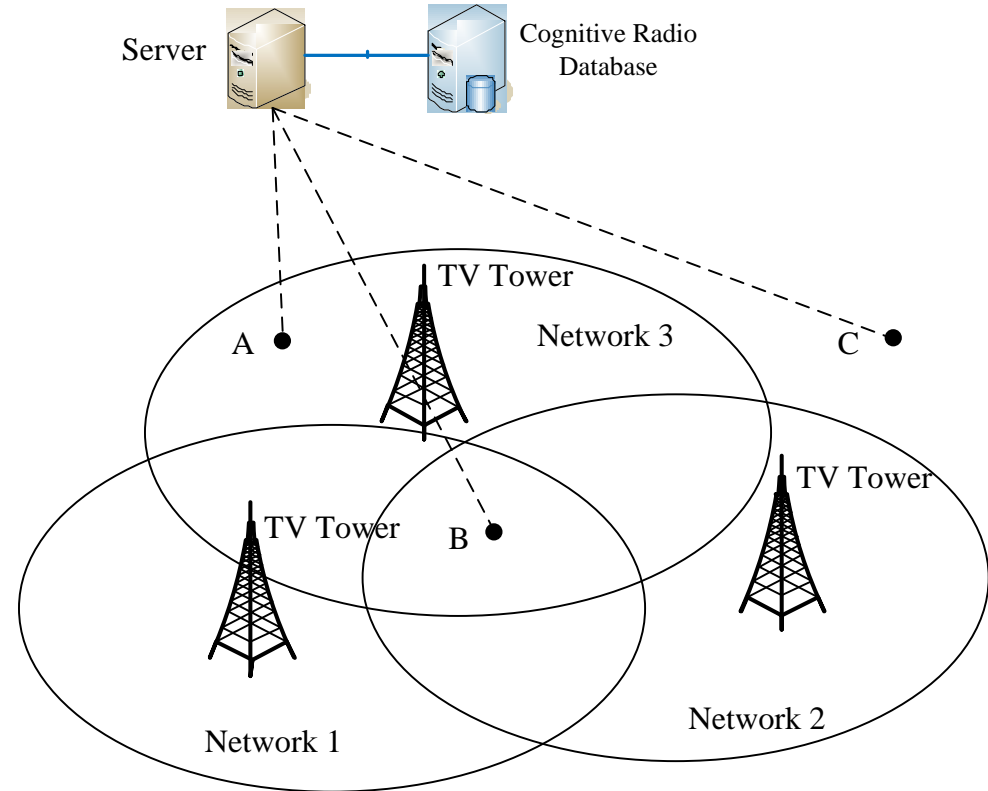

Fig. 1. The geo-location database oriented cognitive radio network 
The information on the spectrum locally available is defined as cognitive information. To deliver the cognitive information, the geographic area covered by geo-location database is divided into small squares called pixels or meshes (where the term "mesh" is adopted in this paper), which is the minimum granularity of cognitive information. The cognitive information of the majority area of a mesh is defined as the cognitive information of the mesh, which is also defined in our previous work [11][12].

Denote the transmit power of TV network as $P_{0}$. Only the path loss is considered. Hence the received power is $P_{r}=P_{0} \frac{V}{L^{\alpha}}$, where $\alpha$ is path-loss factor and $V$ is a frequency dependent constant. We normalize $V$ to be 1 for simplicity and consider $\alpha \geq 2$ which is typical in practice [27]. Define a received power threshold $\eta$. When $P_{r} \leq \eta$, we get the distance $L \geq\left(\frac{\eta}{P_{0} V}\right)^{1 / \alpha}=L_{t h}$, where $L_{t h}$ is the coverage radius of the networks. In order to model more complex network coverage, we further consider the ellipse coverage and irregular network boundaries. It is noted that we have abstract the parameters in the wireless system as a single parameter, namely, the coverage area of a network. Once the coverage of network is achieved, the problem becomes a geometric problem. Then we separate the regions with pure radio environment and approximate them with polygons.

\subsection{Delivery of Cognitive Information}

Unlicensed user can get cognitive information through broadcasting or on-demand method. In broadcasting method, the database broadcasts the cognitive information of each mesh, which contains the geographic information of the mesh in the head of the cognitive information. Once an unlicensed user receives cognitive information, it decodes the head of cognitive information to obtain the geographic information of the mesh where it stays. Then the unlicensed user decides whether it is located in the mesh via positioning method such as GPS (Global Positioning System). If the unlicensed user is located in this mesh, it decodes the remaining cognitive information. Otherwise, it abandons the cognitive information.

In on-demand method, unlicensed user queries database for cognitive information of the mesh where it stays by providing its geographic information. Then the database sends the cognitive information of the mesh to the unlicensed user. The unlicensed user does not need to query the database again as long as it stays inside this mesh. However, once the unlicensed user moves out of the mesh, it needs to query database for new cognitive information.

\subsection{Region Positioning}

We define Region Positioning as to find a method to depict a region. Once a point's geographic information is given, whether or not this point is in the region is also known.

Obviously, rectangle can be used in region positioning. The coordinates of vertexes on diagonal are denoted as $\left(x_{1}, y_{1}\right),\left(x_{2}, y_{2}\right)$ and one point's coordinates are $p(x, y)$. With each coordinate obtained by GPS, the relation between point $p$ and the rectangle is as follows.

$$
p \text { is }\left\{\begin{array}{l}
\text { inside rectangle, } \\
\text { outside rectangle, } \\
x_{1}<x<x_{2} \text { and } y_{1}<y<y_{2}
\end{array}\right.
$$


In this paper, polygon is used in region positioning. The region positioning algorithm with polygon mesh is described in Algorithm 1, where a polygon with $n$ vertexes is stored in an array $P$ that consists of the coordinates of each vertex. $P$ is described as follows.

$$
P=\left[\begin{array}{lllll}
x_{1} & x_{2} & \ldots & x_{n} & x_{1} \\
y_{2} & y_{2} & \ldots & y_{n} & y_{1}
\end{array}\right]
$$

The $i$ th edge of the polygon is denoted as $e_{i}=\{P(:, i), P(:,(i \bmod n)+1)\}$. Notice that Algorithm 1 is a common angorithm in the area of Computational Geometry [26].

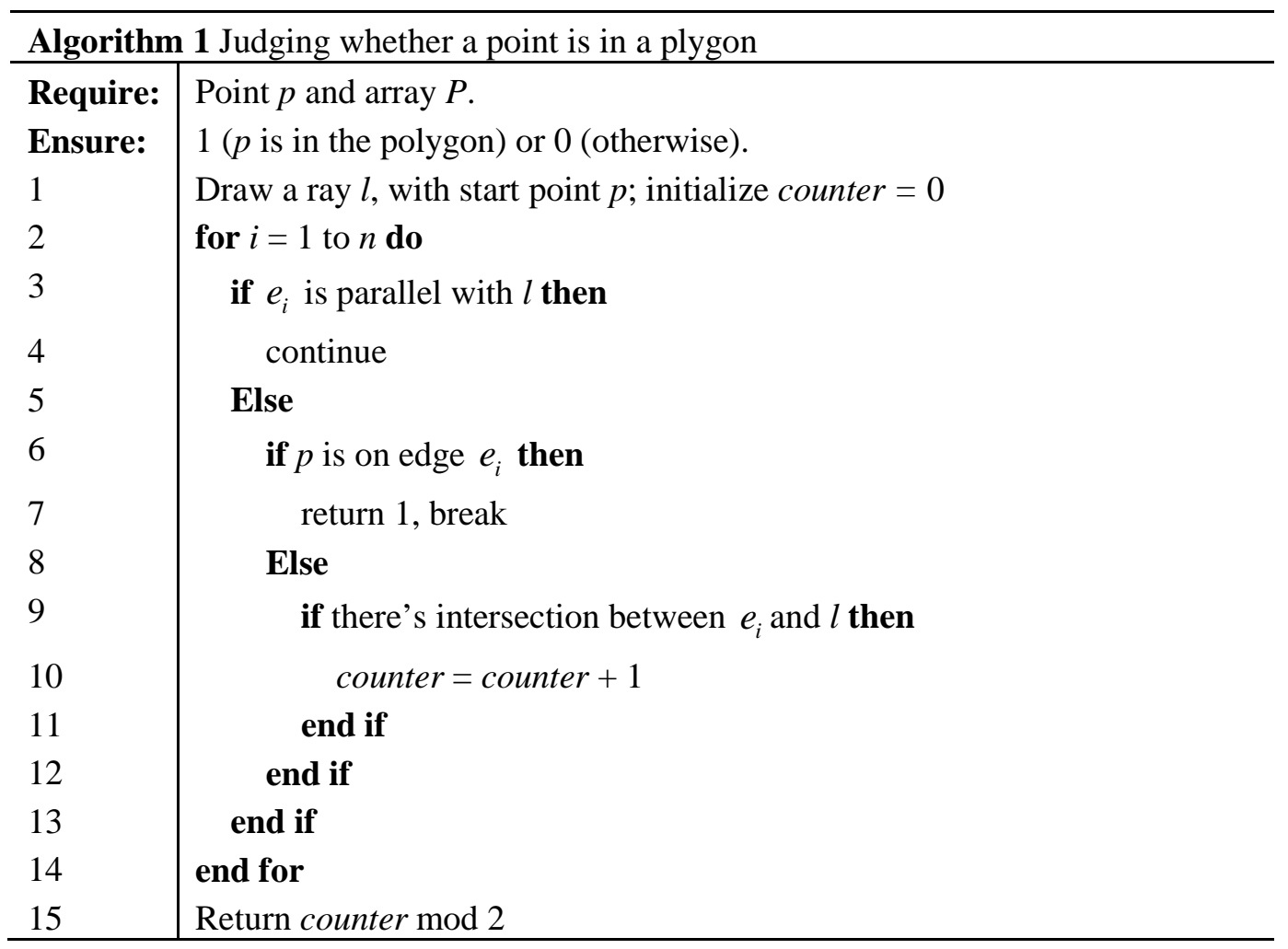

In this paper, polygon based region positioning scheme is addressed. Polygons are used as meshes to cover the entire area. Thus the problem is equivalent to how to divide the entire area into polygons, such that each polygon has almost pure radio environment.

In Fig. 2, there are three TV networks, which divide the entire area into seven regions, i.e. $R_{1}, R_{2}, \ldots, R_{7}$. Thus the radio parameters of each region differ from each other. The following work is to use polygon to approximate regions $R_{i}, i=1,2, \ldots, 7$. 


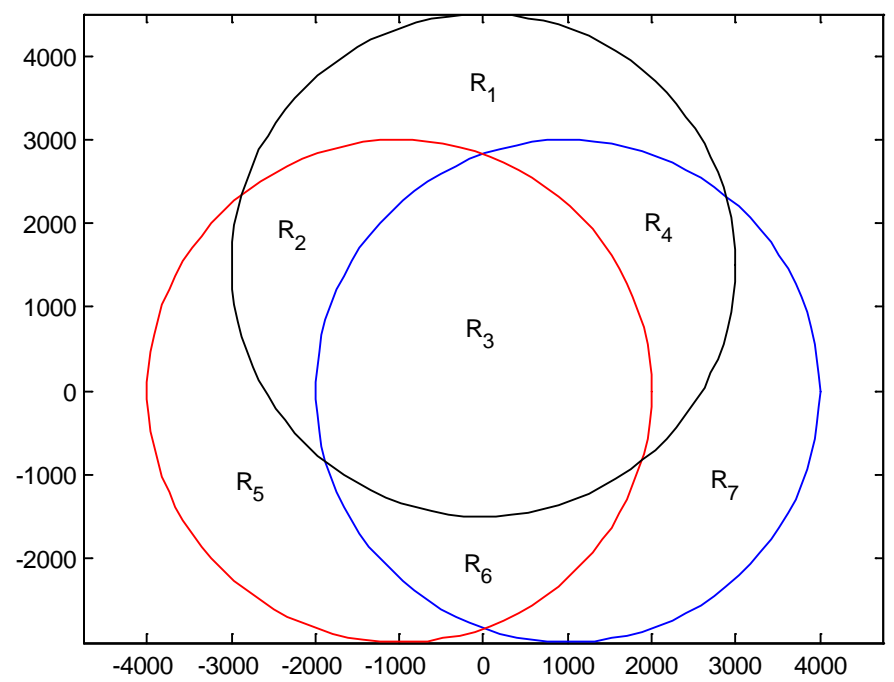

Fig. 2. The deployment of TV networks.

\section{Polygon Approximation Algorithm}

Regions $R_{1}, R_{2}, \ldots, R_{7}$ are called meshes in this paper. The key problems that need to be solved are how to determine one edge of polygon, how to search one polygon (mesh) and how to search all the meshes. They are solved in the following subsections.

\subsection{Determine one edge of polygon}

With the boundary of each network given, the problem is to approximate closed boundary curve with polygon. Local curvature of the boundary curve can be used to determine one edge of polygon. On the part of the curve where the curvature is larger, shorter edge is used to approximate the curve. Otherwise, longer edge is used to approximate the curve. However, this technique strongly relies on the boundary information. On the part of the boundary where the curve is extremely irregular, the performance of this scheme is not ideal. In our work, lower limit error ( $L L E)$ is used to determine one edge. When the error firstly exceeds $L L E$, the end of this edge is determined. The $L L E$ is illustrated in Fig. 3, where the average error of approximation is the margin between the boundary curve and polygon edge, which is formulated as follows.

$$
\text { error }=E\left(\sum_{i} d_{i}\right)
$$


where $E(*)$ is the expectation of “*”. The algorithm is named as Edge Searching Algorithm (ESA), which is described in Algorithm 2.
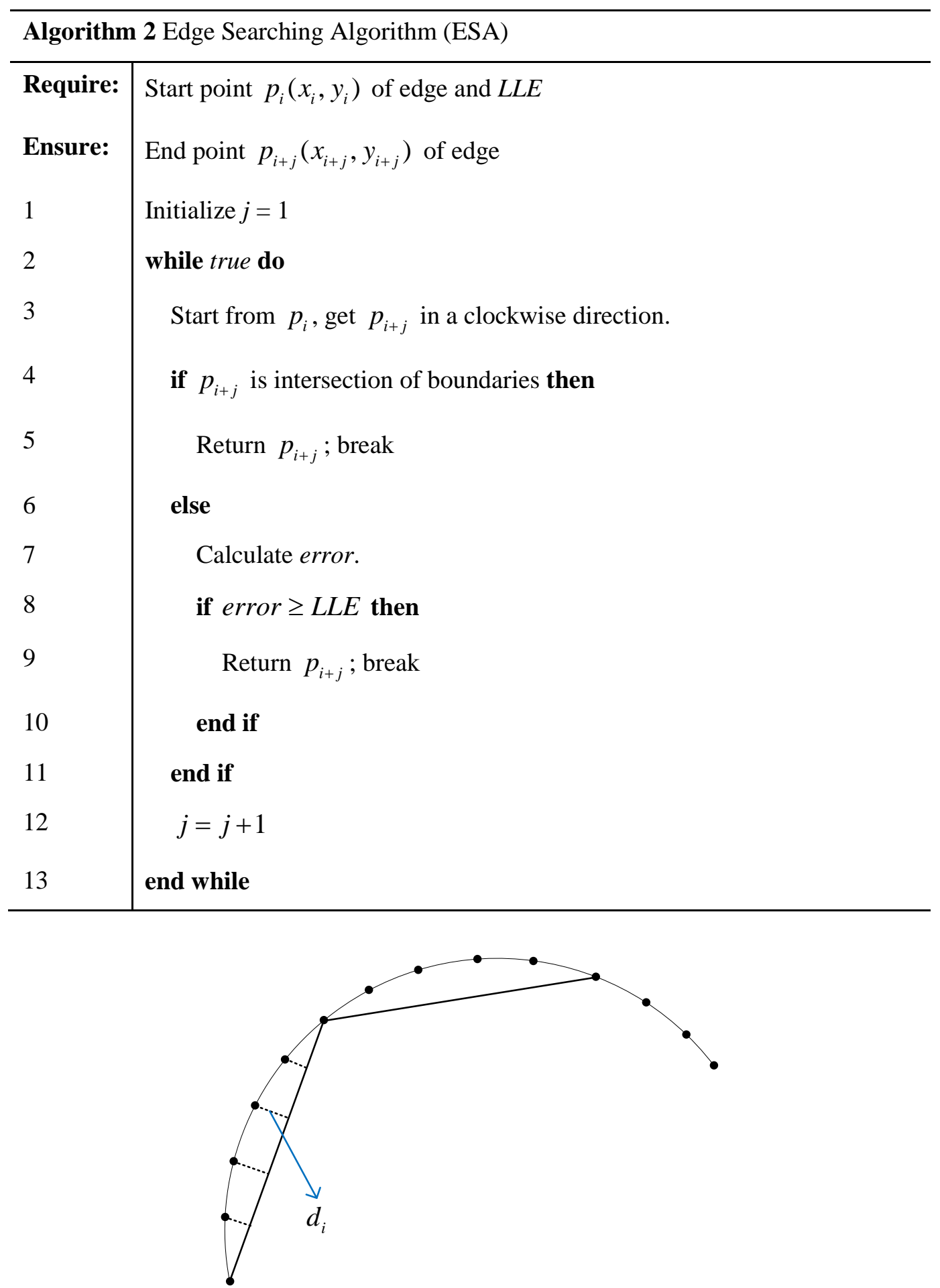

Fig. 3. The method to determine one edge of polygon 


\subsection{Search one polygon (mesh)}

Searching one mesh is a path selection problem at the intersection. As illustrated in Fig. 4, in the intersection $\mathrm{P}$, there is more than one path to choose. Path $\overline{P A}$ is the extended line of $\overline{O P}$. Paths $\overline{P B}$ and $\overline{P C}$ are the tangents of the boundaries of each network. Obviously, $\overline{P C}$ is the correct path. Generally, the path that has the maximum angle between original path $(\overline{P A})$ in clockwise direction is the correct path. Thus with the direction of chosen path, the end point of polygon's edge can also be found.

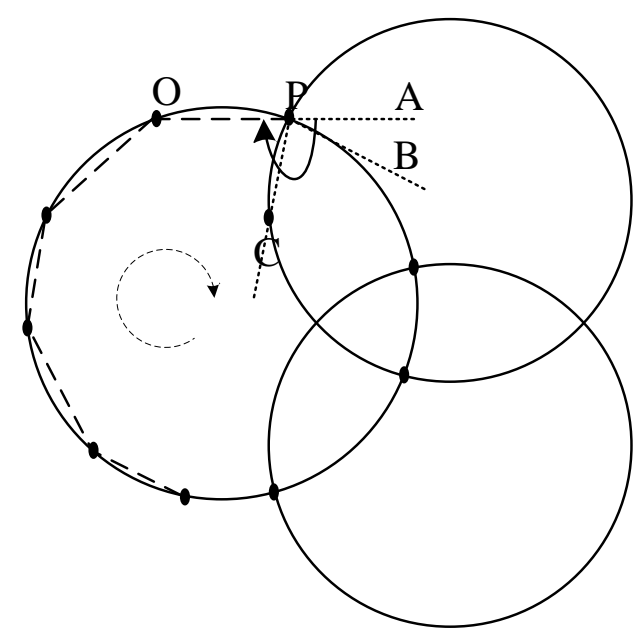

Fig. 4. The method to search one polygon

The cross product of vectors is adopted to determine the turning direction of one segment versus another segment. The dot product of vectors is used to estimate the angle between two segments. Denote two segments $l_{1}=\left[p_{i}, p_{j}\right]$ and $l_{2}=\left[p_{j}, p_{k}\right]$ with point $p_{j}$ in common, where $\left[p_{m}, p_{n}\right]$ is a segment with start point $p_{m}$ and end point $p_{n}$. For $a=l_{1} \times l_{2}$, we have conclusions as follows.

- If $a<0$, then by rotating $l_{1}$ around $p_{j}$ in counterclockwise direction, $l_{1}$ can finally coincides with $l_{2}$.

- If $a=0$, then $p_{i}, p_{j}$ and $p_{k}$ are collinear.

- If $a>0$, then by rotating $l_{1}$ around $p_{j}$ in clockwise direction, $l_{1}$ can finally coincides with $l_{2}$. 
Define angle $\theta=\left\langle l_{1}, l_{2}\right\rangle=\arccos \frac{\left(p_{k}-p_{j}\right) \cdot\left(p_{j}-p_{i}\right)}{\left\|p_{k}-p_{j}\right\| \cdot\left\|p_{j}-p_{i}\right\|} \in[0, \pi]$. With $a$ and $\theta$ known, path selection algorithm (PSA) is described in Algorithm 3.

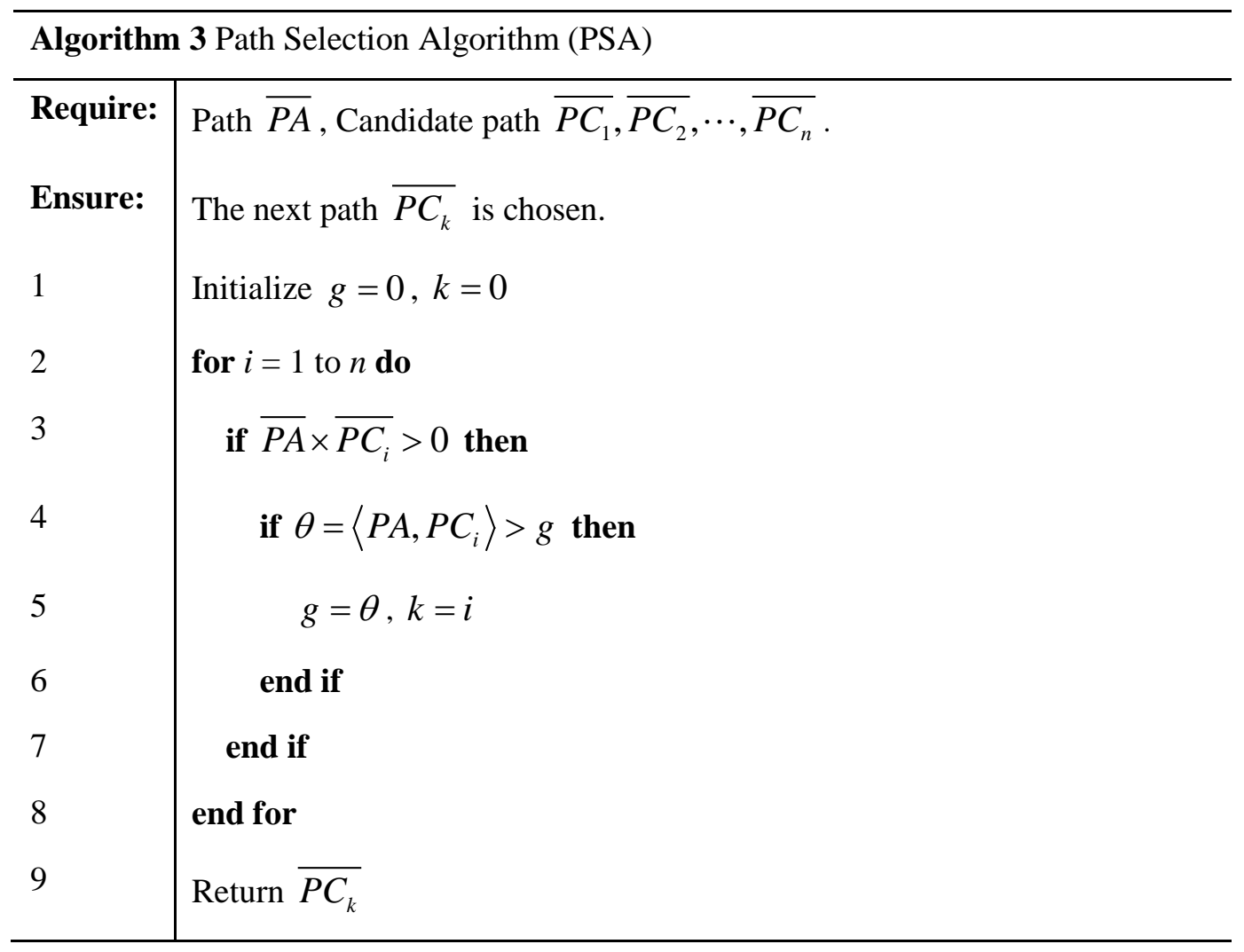

\subsection{Search all the meshes}

Firstly, the data structure of the intersection of two networks (denoted as Network A and Network B) is presented in Table 1.

- $P_{A, L}\left(X_{A, L}, Y_{A, L}\right), P_{A, R}\left(X_{A, R}, Y_{A, R}\right):$ Coordinates of two vertexes that are adjacent with $P$ in the boundary of network A.

- $P_{B, L}\left(X_{B, L}, Y_{B, L}\right), P_{B, R}\left(X_{B, R}, Y_{B, R}\right)$ : Coordinates of two vertexes that are adjacent with $P$ in the boundary of network B.

- $I_{A, L}, I_{A, R}, I_{B, L}, I_{B, R}$ : Access labels of $P$ in four directions: $P_{A, L} \rightarrow P, P_{A, R} \rightarrow P$, $P_{B, L} \rightarrow P, P_{B, R} \rightarrow P$. Define $I_{P_{s}, P}$ (the access label of $P$ in the direction $P_{s} \rightarrow P$ ) as follows. 


$$
I_{P_{s}, P}=\left\{\begin{array}{l}
0, \text { get in } P \text { from } P_{s}, \text { i.e., access negatively } \\
1, \text { get out of } P \text { to } P_{s}, \text { i.e., access positively } \\
2, P \text { is not accessed }
\end{array}\right.
$$

Table 1. Data structure of the intersection of two networks

\begin{tabular}{|l|l|l|l|l|l|l|}
\hline Network A & $X_{A, L}$ & $X_{A, R}$ & $X_{B, L}$ & $X_{B, R}$ & $I_{A, L}$ & $I_{B, L}$ \\
\hline Network B & $Y_{A, L}$ & $Y_{A, R}$ & $Y_{B, L}$ & $Y_{B, R}$ & $I_{A, R}$ & $I_{B, R}$ \\
\hline
\end{tabular}

The algorithm that searches all the meshes is described in Algorithm 4, which is denoted as MSA.

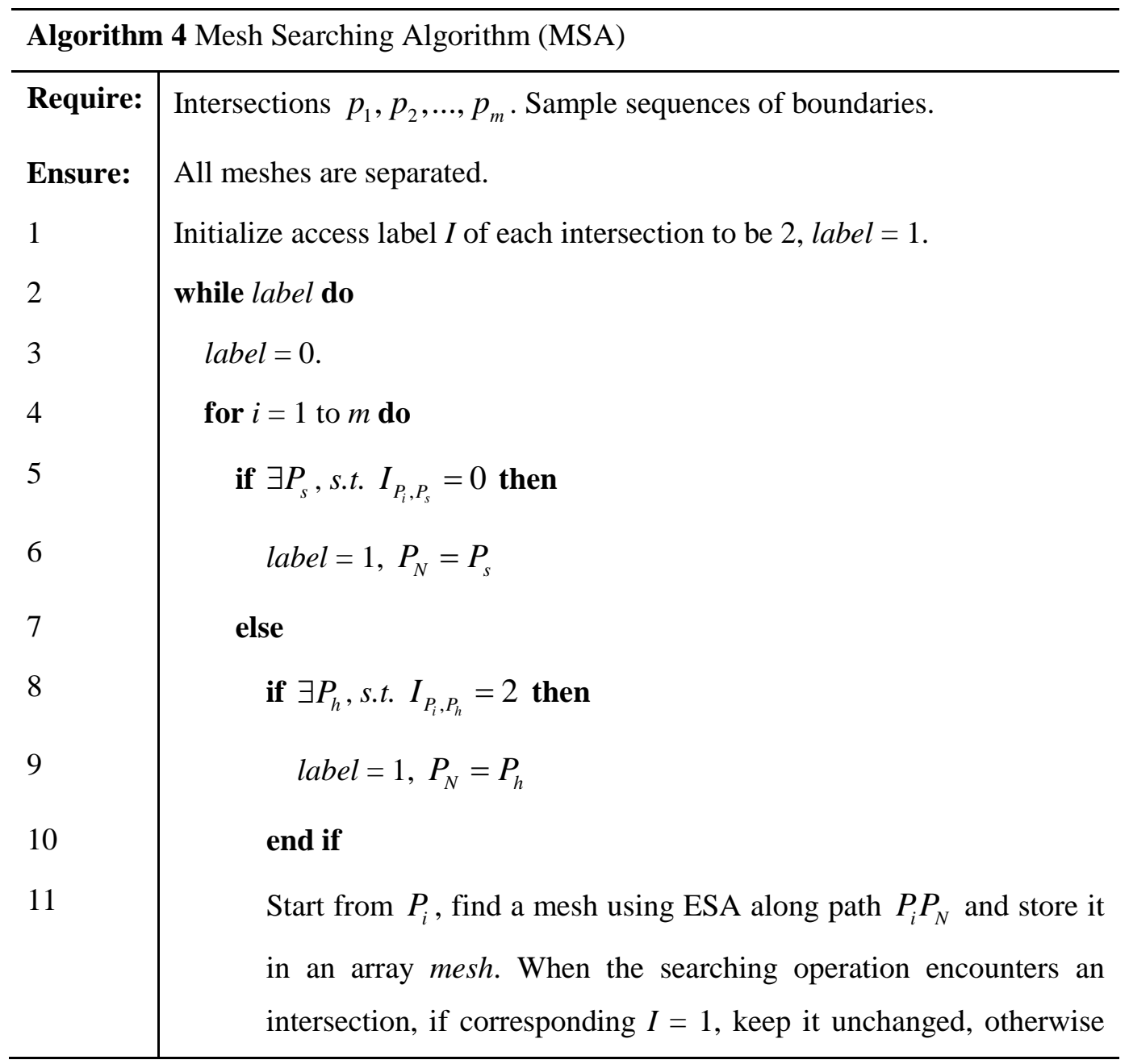




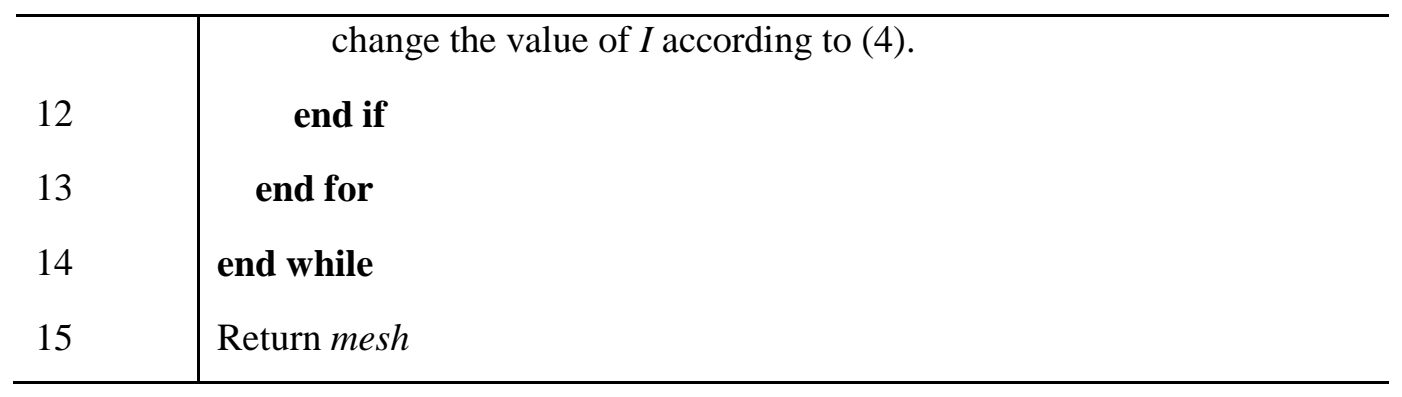

The correctness of algorithm MSA is verified by the following theorem.

\section{Theorem 1. All meshes can be found using the algorithm MSA.}

Proof: MSA can guarantee that each intersection can be accessed positively in each direction. As illustrated in Fig. 5, take intersection $P_{A}$ for example, MSA can guarantee that $P_{A}$ is accessed positively in the direction $P_{N_{1}, L} \rightarrow P_{A}$. We use the technique of reduction to absurdity in the proof of Theorem 1.

Assume that mesh $R_{5}$ is not found, then $P_{A}$ is not accessed in the direction $P_{A} \rightarrow P_{N_{2}, L}$ either. Thus $I_{P_{N_{2}, L}, P_{A}} \neq 1$, which is contradictory to the results of MSA. Therefore the correctness of MSA is proved by this contradiction.

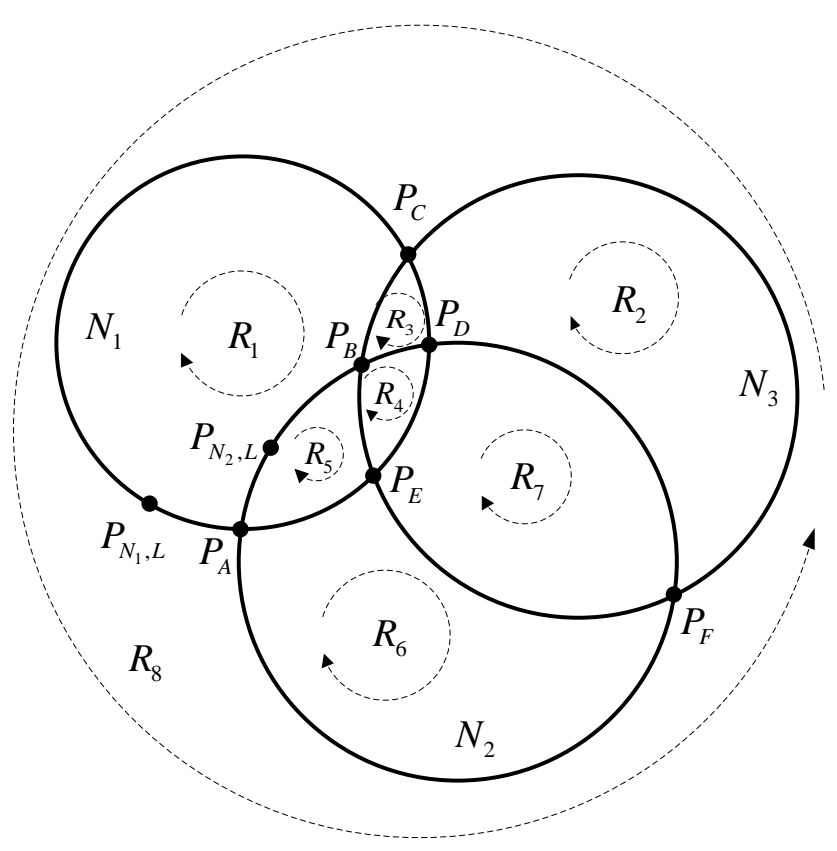

Fig. 5. All meshes can be found 


\subsection{Overall algorithm of polygon approximation}

Overall algorithm is described in Algorithm 5. The first step is the basic segment approximation. Then we need to find the intersections and adjust them into the vertex sequence of polygon mesh. After finding all the meshes, the overall algorithm is finished.

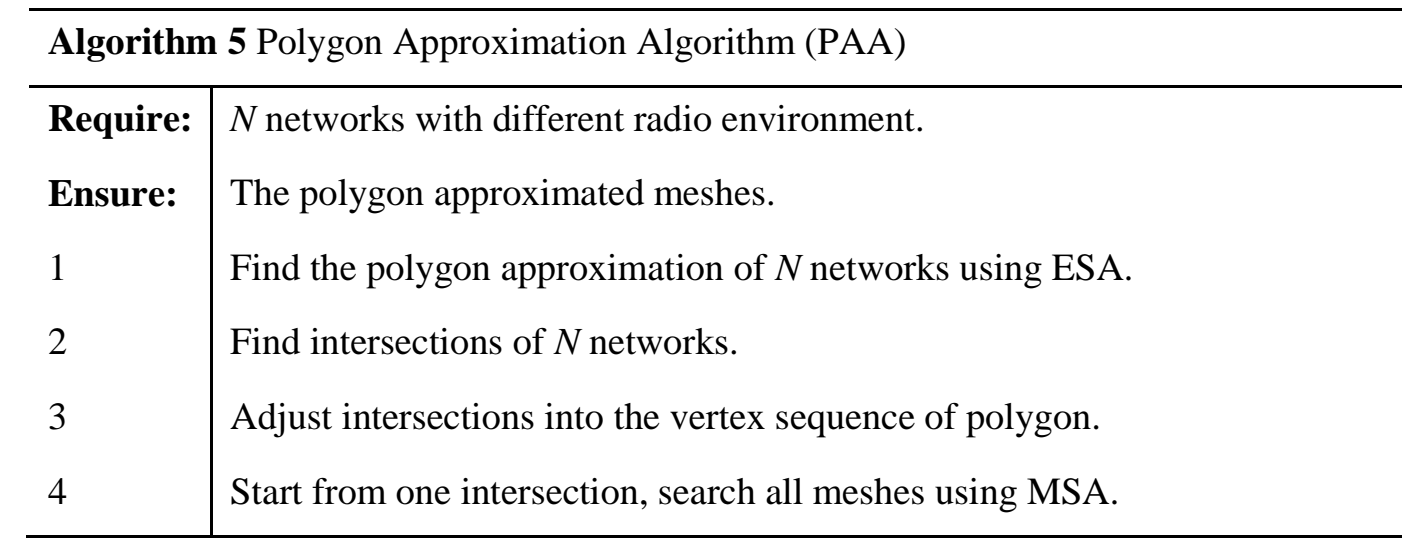

The key idea of our algorithm is to guarantee that each intersection is accessed positively in each direction. Thus each region with pure radio environment is separated. The basic operation of Algorithm 5 is to use polygons to approximate each region with pure radio environment. Thus the cognitive information can be classified and stored with polygon regions. When the cognitive information needs to be delivered, it can be delivered with the cognitive information of polygon, which can cover a large area. Thus the error and efficiency can be guaranteed simultaneously. Further, we have addressed the error of cognitive information in the next section.

\section{Analysis of Polygon Approximation Algorithm}

In polygon approximation algorithm, the error of cognitive information along the $j$ th edge of the polygon is the area of the shaded region in Fig. 6. The relation between the error of cognitive information and the number of edges of polygon is provided in Theorem 2.

Theorem 2. The relation between the error of cognitive information $\varepsilon$ and the number of edges of the polygon $m$ is

$$
\varepsilon=\Omega\left(\frac{1}{m^{2}}\right)
$$

Proof: In Fig. 6, the error of cognitive information, namely, the area of the shaded region is as 
follows.

$$
\varepsilon=\sum_{i=1}^{m}\left(\frac{\alpha_{i} R^{2}}{2}-\frac{R^{2} \sin \frac{\alpha_{i}}{2} \sqrt{1-\left(\sin \frac{\alpha_{i}}{2}\right)^{2}}}{2}\right)=\sum_{i=1}^{m} f\left(\alpha_{i}\right)
$$

where $f\left(\alpha_{i}\right)=\frac{\alpha_{i} R^{2}}{2}-R^{2} \sin \frac{\alpha_{i}}{2} \sqrt{1-\left(\sin \frac{\alpha_{i}}{2}\right)^{2}}$. The third-order Taylor series of $f\left(\alpha_{i}\right)$ at the point $\alpha_{i}=0$ is

$$
f\left(\alpha_{i}\right)=R^{2}\left(\frac{\alpha_{i}^{3}}{12}+o\left(\alpha_{i}^{4}\right)\right)
$$

When $m \rightarrow \infty, \alpha_{i} \rightarrow 0$. The term of $o\left(\alpha_{i}^{4}\right)$ can be omitted, then we have

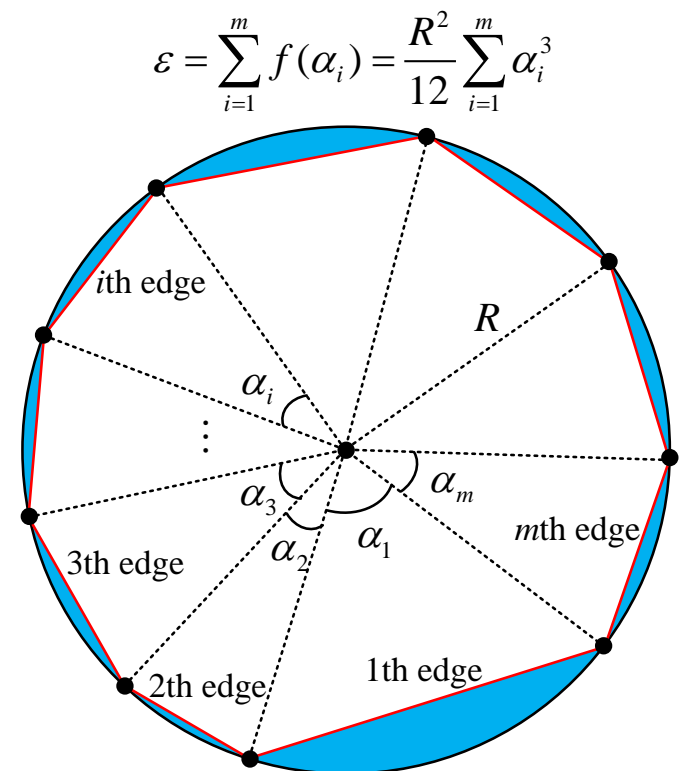

Fig. 6. The polygon approximation

Using the Hölder's inequality and letting $p=3, q=3$ / 2 , we have

$$
\left(\sum_{i=1}^{m} \alpha_{i}^{3}\right)^{1 / 3}\left(\sum_{i=1}^{m} 1^{3}\right)^{2 / 3} \geq \sum_{i=1}^{m}\left|a_{i} * 1\right|=2 \pi
$$

Therefore, we have

$$
\sum_{i=1}^{m} \alpha_{i}^{3} \geq \frac{8 \pi^{3}}{m^{2}}
$$

The lower bound of error of cognitive information is

$$
\varepsilon=\sum_{i=1}^{m} f\left(\alpha_{i}\right) \geq \frac{2 R^{2} \pi^{3}}{3} \frac{1}{m^{2}}
$$

The equality is achieved when $\alpha_{1}=\alpha_{2}=\ldots=\alpha_{m}$. Thus the theorem is proved. 
Lemma 1. (Hölder's inequality, [28]) For the n-dimensional Euclidean space, when the set $S$ is $\{1, \ldots, n\}$ with the counting measure, we have

$$
\sum_{k=1}^{n}\left|x_{k} y_{k}\right| \leq\left(\sum_{k=1}^{n}\left|x_{k}\right|^{p}\right)^{1 / p}\left(\sum_{k=1}^{n}\left|y_{k}\right|^{q}\right)^{1 / q}, \forall\left(x_{1}, \cdots, x_{n}\right),\left(y_{1}, \cdots, y_{n}\right) \in \mathbb{R}^{n} \text { or } \mathbb{C}^{n}
$$

where $p, q \in[1,+\infty)$ with $\frac{1}{p}+\frac{1}{q}=1$. The equality is achieved when $c_{1}\left|x_{k}\right|^{p}=c_{2}\left|y_{k}\right|^{q}$ with $c_{1} \geq 0, c_{2} \geq 0, c_{1} c_{2} \neq 0$.

Notice that the number of edges determines the complexity of the polygon approximation algorithm. Theorem 2 has verified that the error of cognitive information will decrease with the increase of the number of edges. In practice, if the computation complexity can be afforded, the number of edges can be increased to minimize the error. Hence there is a tradeoff between the complexity of algorithm and accuracy of the cognitive information.

\section{Numerical Results and Analysis}

In this section, the polygon approximation algorithm is simulated. Firstly we provide the scenario where three TV networks exist. For simplicity, the coverage area of TV network is circle. As illustrated in Fig. 7(a), there is overlapped area among these TV networks. In Fig. 7(b), we have provided another scenario with three TV networks, where the shape of the TV network is ellipse. The parameters for simulations are provided in Table 2.

Table 2. Parameters in simulation

\begin{tabular}{|l|c|}
\hline \multicolumn{1}{|c|}{ Parameter } & Value \\
\hline Radius of network coverage for different circle networks & $3 \mathrm{~km}$ and $2 \mathrm{~km}$ \\
\hline Error threshold for circle networks & $1.2 \mathrm{~m}$ \\
\hline Long radius for different ellipse networks & $5 \mathrm{~km}$ and 4km \\
\hline Short radius for different ellipse networks & $2 \mathrm{~km}$ \\
\hline Error threshold for ellipse networks & $2 \mathrm{~m}$ \\
\hline
\end{tabular}

In Fig. 8(a), the polygon approximation results are achieved, where the regions $R_{i}(\mathrm{i}=1,2, \ldots, 7)$ in Fig. 7(a) are separated. In Fig. 8(b), the regions $R_{i}(\mathrm{i}=1,2, \ldots, 9)$ are separated. Notice that each region is separated accurately. However, when the cognitive information is delivered, the geographic information of each edge of a polygon must be delivered. If the bandwidth to deliver the cognitive information is limited, we need to triangulate the polygons. The Delaunay triangulation results are illustrated in Fig. 9(a) and Fig. 9(b), which are the triangulation results of the polygons in Fig. 7(a) and Fig. 7(b) respectively. When polygons are triangulated, the cognitive information is much less. Because the shape of region is triangle, the number of regions are larger compared with the polygon region. Therefore there is a tradeoff between the efficiency and bandwidth in the delivery of cognitive information. 


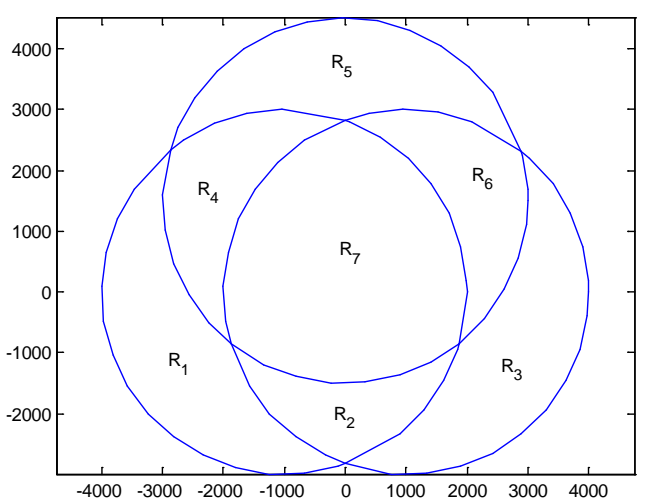

Fig. 7(a). The scenario where the shape of TV network is circle.
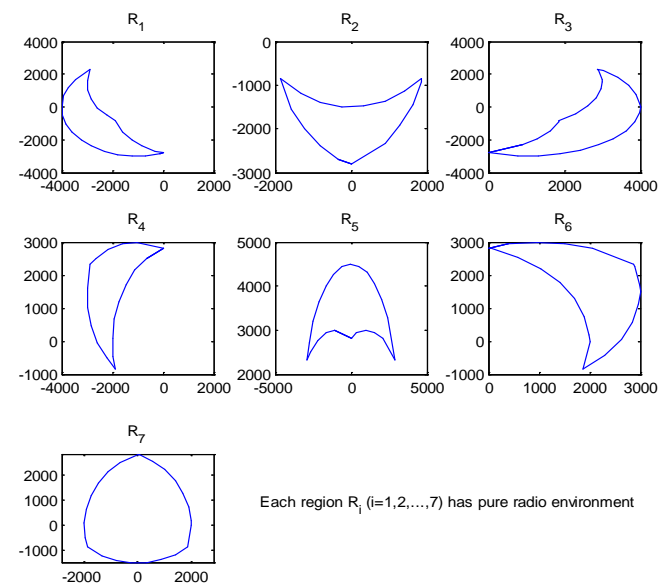

Each region $R_{i}(i=1,2, \ldots, 7)$ has pure radio enuronment

Fig. 8(a). The polygon approximation results of Fig. 7(a), where each region is separated.

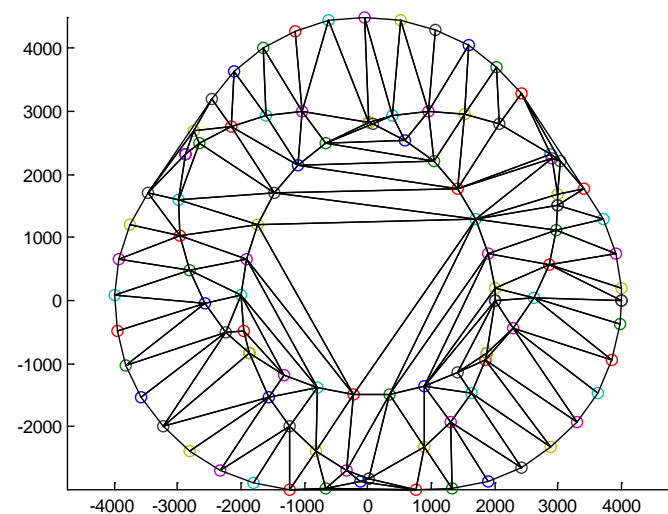

Fig. 9(a). The Delaunay triangulation results of the polygons in Fig. 7(a).

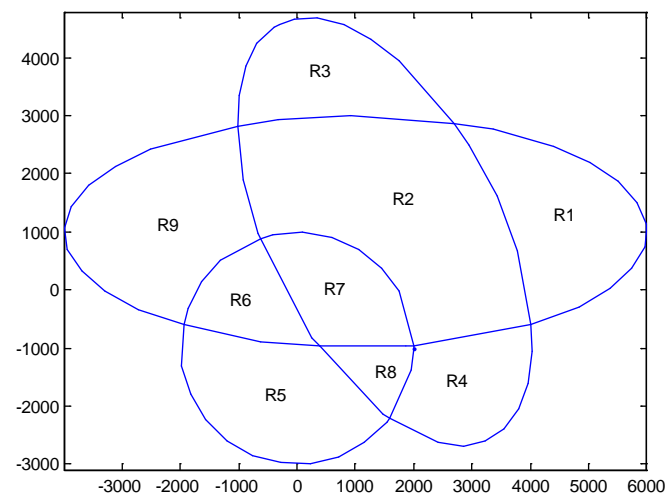

Fig. 7(b). The scenario where the shape of TV network is ellipse.
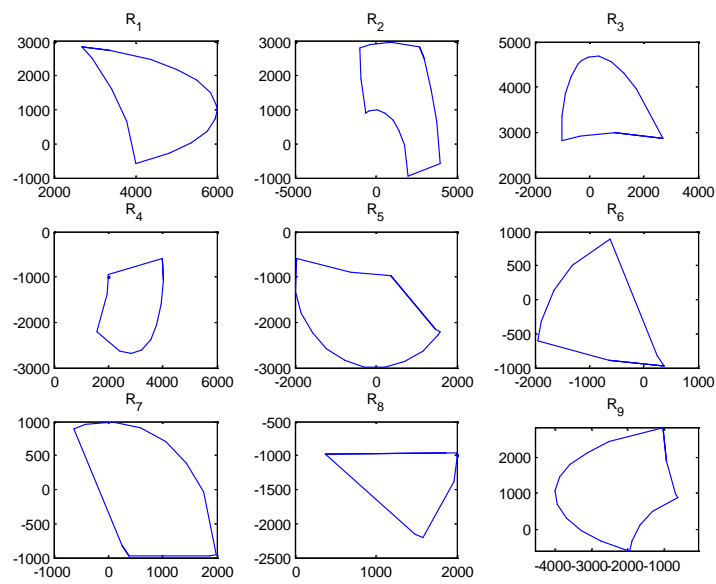

Fig. 8(b). The polygon approximation results of

Fig. 7(b), where each region is separated.

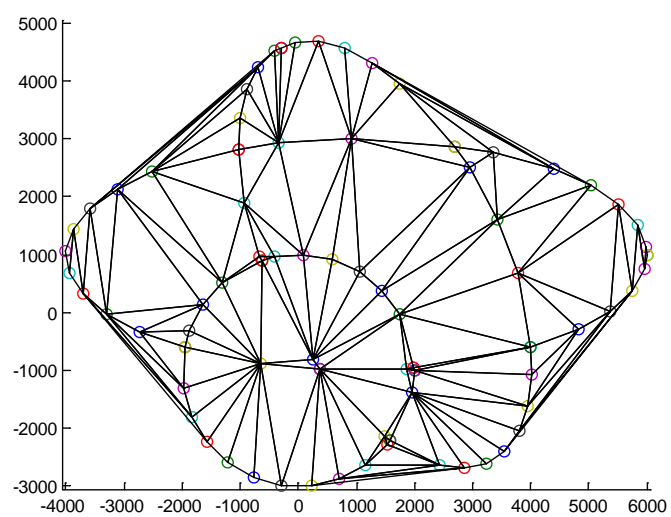

Fig. 9(b). The Delaunay triangulation results of the polygons in Fig. 7(b). 
Since the number of polygons' edge has an impact on the efficiency of cognitive information delivery, we have provided the relation between the number of polygons' edges $m$ and the error of cognitive information $\varepsilon$ in Fig. 10, where the error is defined in (3). It is noted that with the increase of $m$, the error $\varepsilon$ is decreasing, which is obvious since when $m$ is increasing, the polygons will approximate the boundaries of networks and the error will decrease. In Theorem 2, we have achieved the relation between the error $\varepsilon$ and the number of polygons' edge $m$ as $\varepsilon=\Omega\left(\frac{1}{m^{2}}\right)$. In Fig. 11, we have verified this relationship by data fitting. The relation between $\varepsilon$ and $\frac{1}{m^{2}}$ is plotted in Fig. 11. Besides, the data fitting shows that with the ellipse network, the relation between $\varepsilon$ and $\frac{1}{\mathrm{~m}^{2}}$ is as follows.

$$
\varepsilon=\frac{1.7165 \times 10^{4}}{m^{2}}
$$

With the ellipse network, the relation between $\varepsilon$ and $\frac{1}{\mathrm{~m}^{2}}$ is as follows.

$$
\varepsilon=\frac{2.407 \times 10^{4}}{m^{2}}
$$

which has verified the relation $\varepsilon=\Omega\left(\frac{1}{\mathrm{~m}^{2}}\right)$.

Besides, the curve with circle network is above the curve with ellipse network in Fig. 10. Because the average curvature of circle is larger than that of ellipse, the error with circle network is also larger than the error with ellipse network for the same number of edges.

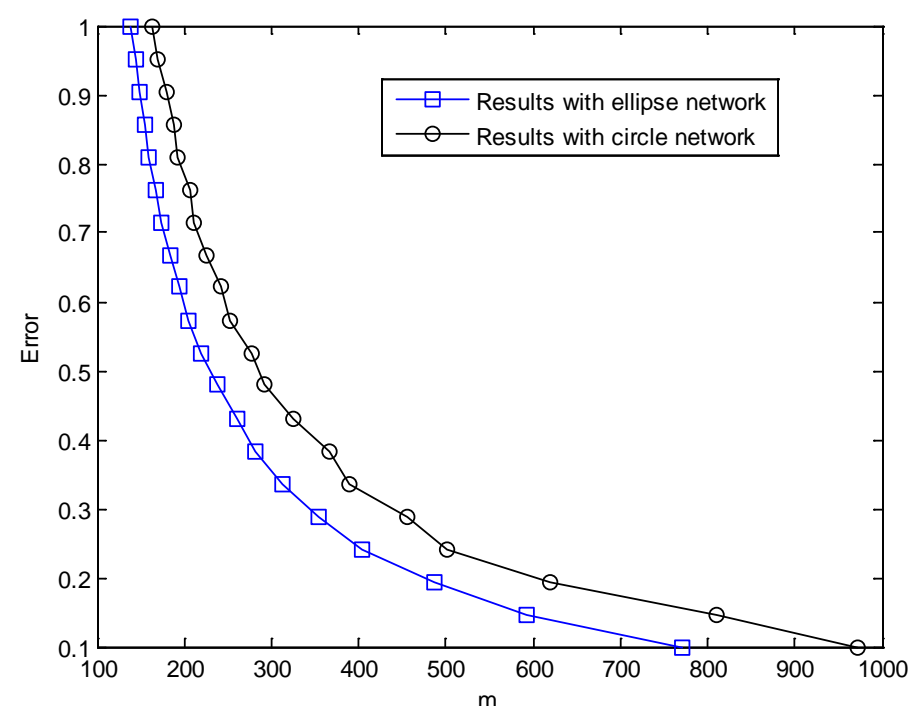

Fig. 10. The error versus the number of polygons' edges $(m)$. 


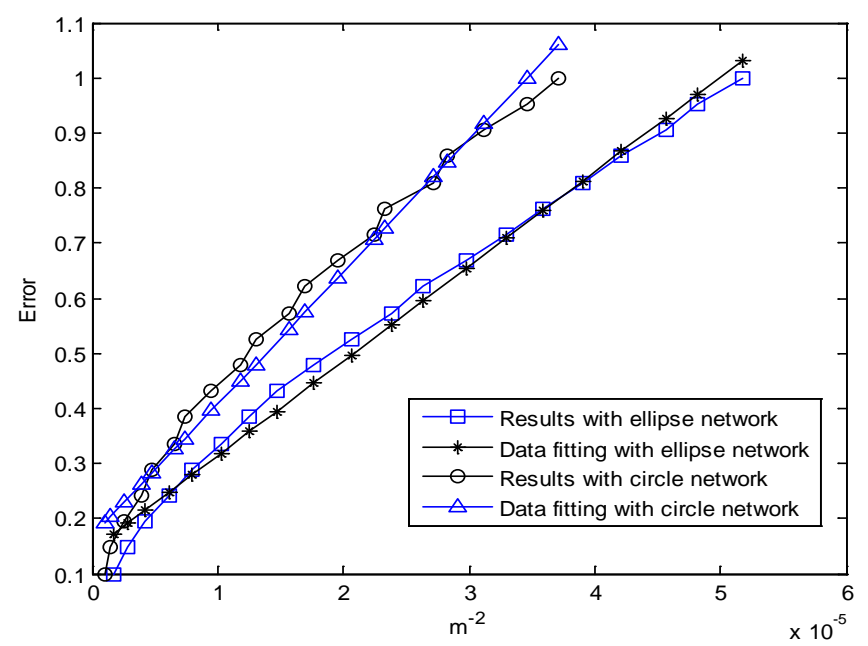

Fig. 11. The data fitting of the relation between error and the number of polygons' edges (m)

Finally, we consider more practical scenarios that the boundaries of networks are irregular, which are illustrated in Fig. 12 and Fig. 13. For the networks in Fig. 12(a) and Fig. 13(a), the polygon approximations are correct. Take Fig. 12 for an example, the regions $R_{1}, R_{2}, \ldots, R_{7}$ in Fig. 12(b) are correctly separated from Fig. 12(a), which is the same for Fig. 13. Hence when the boundaries of networks are irregular, our algorithms can still approximate the boundaries of networks and separate each region from the practical deployment of networks.

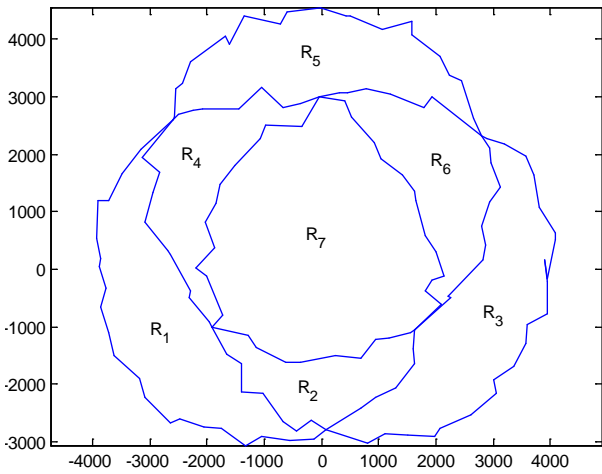

Fig. 12(a). A practical scenario

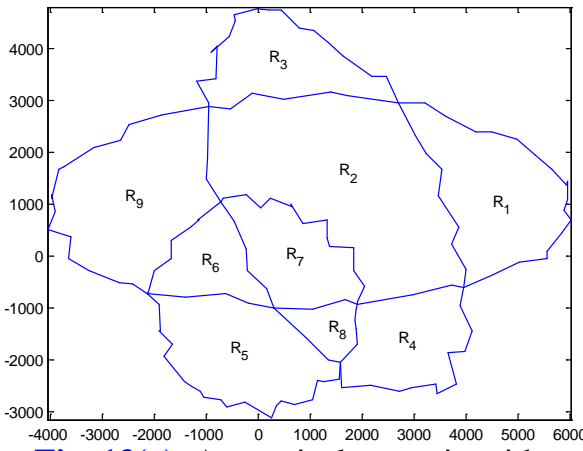

Fig. 13(a). A practical scenario with more complex network deployment

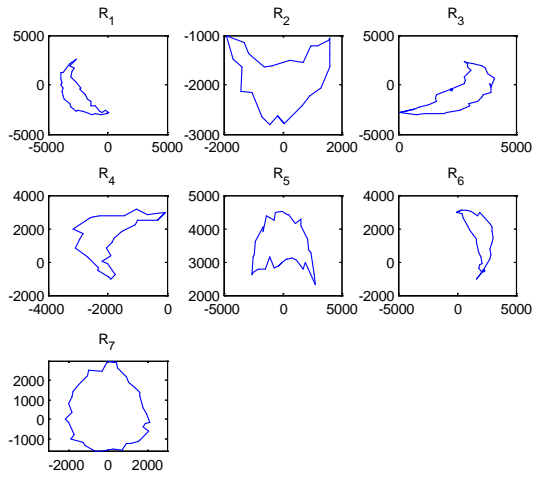

Fig. 12(b). The polygon approximation of Fig. 12(a)
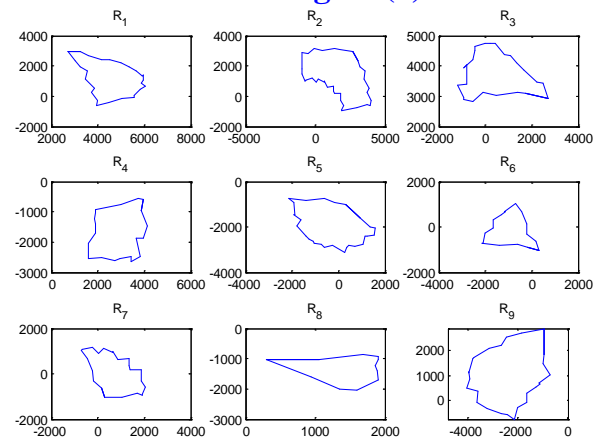

Fig. 13(b). The polygon approximation of

Fig. 13(a) 


\section{Conclusion}

In this paper, we adopt the polygon meshes to classify the cognitive information. Thus the number of meshes can be reduced significantly. Besides, polygon mesh can well approximate the real environment and reduce the error of cognitive information compared with rectangle meshes because the edges of polygon are selected along the boundaries of networks in polygon approximation. We firstly design polygon approximation algorithm. Then we have analyzed the relation between the error of cognitive information and the number of polygon's edges. Finally, we provide the simulation results to validate our algorithm and analysis.

\section{Acknowledgement}

This work is supported by the National Natural Science Foundation of China (No. 61601055, No. 61631003), and the National Science Fund for Distinguished Young Scholars (No. 61525101).

\section{References}

[1] McHenry M. A., "NSF Spectrum Occupancy Measurements Project Summary,” Tech. Rep. of Shared Spectrum Company, 2005.

[2] C. R. Stevenson, G. Chouinard, Z. Lei, W. Hu, S. J. Shellhammer, W. Caldwell, "IEEE 802.22: The First Cognitive Radio Wireless Regional Area Network Standard,” IEEE Communications Magazine, vol. 47, no. 1, pp. 130 - 138, Jan. 2009. Article (CrossRefLink)

[3] N. Tadayon, S. Aïssa, “A Multichannel Spectrum Sensing Fusion Mechanism for Cognitive Radio Networks: Design and Application to IEEE 802.22 WRANs,” IEEE Transactions on Cognitive Communications and Networking, vol. 1, no. 4, pp. 359 - 371, Mar. 2016. Article (CrossRefLink)

[4] K. Illanko, M. Naeem, A. Anpalagan, D. Androutsos, "Energy-Efficient Frequency and Power Allocation for Cognitive Radios in Television Systems,” IEEE Systems Journal, vol. 10, no. 1, pp. 313 - 324, Feb. 2015. Article (CrossRefLink)

[5] Woongsup Lee, Dong-Ho Cho, "Comparison of Channel State Acquisition Schemes in Cognitive Radio Environment,” IEEE Transactions on Wireless Communications, vol. 13, no. 4, pp. 2295 2307, Feb. 2014. Article (CrossRefLink)

[6] H. B. Yilmaz, T. Tugcu, F. Alagöz, S. Bayhan, "Radio Environment Map as Enabler for Practical Cognitive Radio Networks,” IEEE Communications Magazine, vol. 51, no. 12, pp. 162 - 169, Dec. 2013. Article (CrossRefLink)

[7] F. Paisana, N. Marchetti, L. A. DaSilva, "Radar, TV and Cellular Bands: Which Spectrum Access Techniques for Which Bands?” IEEE Communications Surveys \& Tutorials, vol. 16, no. 3, pp. 1193 - 1220, Apr. 2014. Article (CrossRefLink)

[8] FCC. Second Report and Order and Memorandum Opinion and Order: In the Matter of Unlicensed Operation in the TV Broadcast Bands, Doc. 08-260, 2008. Article (CrossRefLink)

[9] FCC. Second Memorandum Opinion and Order, Doc. 10-174, 2010.

[10] ECC. Technical and operational requirements for the operation of white space devices under geo-location approach, ECC report 186, 2013.

[11] Z. Feng, Z. Wei, Q. Zhang, W. Li, et al., "Cognitive Information Delivery in Geo-location Database based Cognitive Radio Networks,” Wireless Communications and Mobile Computing, vol. 16, no. 13, Sep. 2016. Article (CrossRefLink)

[12] Z. Wei, Q. Zhang, Z. Feng, W. Li and T. A. Gulliver, "On the construction of Radio Environment Maps for Cognitive Radio Networks,” in Proc. of IEEE Wireless Communications and Networking Conference (WCNC), pp. 4504-4509, Apr. 2013. Article (CrossRefLink) 
[13] J. Perez-Romero, A. Zalonis, L. Boukhatem, et al., "On the use of radio environment maps for interference management in heterogeneous networks,” IEEE Communications Magazine, vol. 53, no. 8, pp. 184-191, Aug. 2015. Article (CrossRefLink)

[14] Y. Liu, R. Yu, M. Pan, Y. Zhang, S. Xie, "SD-MAC: Spectrum Database-Driven MAC Protocol for Cognitive Machine-to-Machine Networks,” IEEE Transactions on Vehicular Technology, vol. 66, no. 2, pp. 1456-1467, Feb. 2017. Article (CrossRefLink)

[15] M. Caleffi, A. S. Cacciapuoti, “Optimal Database Access for TV White Space,” IEEE Transactions on Communications, vol. 64, no. 1, pp. 83 - 93, Nov. 2015. Article (CrossRefLink)

[16] FCC. Second Report and Order and Memorandum Opinion and Order: In the Matter of Unlicensed Operation in the TV Broadcast Bands, Doc. 08-260, 2008.

[17] FCC. SecondMemorandum Opinion and Order, Doc. 10-174, 2010.

[18] Q. Zhang, Z. Feng, G. Zhang, Q. Li, and P. Zhang, "Efficient Mesh Division and Differential Information Coding Schemes in Broadcast Cognitive Pilot Channel," Wireless Personal Communications, vol. 63, no. 2, pp. 363-392, 2012. Article (CrossRefLink)

[19] ECC. Technical and operational requirements for the operation of white space devices under geo-location approach, ECC report 186, 2013.

[20] C-S. Sum, H. Harada, F. Kojima and etc., "Smart Utility Networks in TV White Space,” IEEE Communications Magazine, vol. 49, no. 7, pp. 132-139, 2011. Article (CrossRefLink)

[21] M. Barrie, S. Delaere, P. Anker and P. Ballon, "Aligning technology, business and regulatory scenarios for cognitive radio,” Telecommunications Policy, vol. 36, no. 7, pp. 546-559, 2012. Article (CrossRefLink)

[22] Z. Feng, Z. Wei, Q. Zhang, and P. Zhang, "Fractal theory based dynamic mesh grouping scheme for efficient cognitive pilot channel design,” Chinese Science Bulletin, vol. 57, no. 28-29, pp. 3684-3690, Sep. 2012. Article (CrossRefLink)

[23] H. Braham; S. Ben Jemaa; G. Fort; E. Moulines; B. Sayrac, "Fixed Rank Kriging for Cellular Coverage Analysis,” IEEE Transactions on Vehicular Technology, Online Publication, Aug. 2016. Article (CrossRefLink)

[24] H. Zou, Ming Jin, H. Jiang, L. Xie and C. Spanos, "WinIPS: WiFi-based non-intrusive IPS for online radio map construction," in Proc. of IEEE Conference on Computer Communications Workshops (INFOCOM WKSHPS), pp. 1081-1082, Apr. 2016. Article (CrossRefLink)

[25] S. Sodagari, “A Secure Radio Environment Map Database to Share Spectrum,” IEEE Journal of Selected Topics in Signal Processing, vol. 9, no. 7, pp. 1298-1305, Oct. 2015. Article (CrossRefLink)

[26] Peide Zhou, “Computational Geometry: Algorithm Design and Analysis,” Tsinghua University Press, Sep. 2011.

[27] Z. Wei, Z. Feng, Q. Zhang and W. Li, "Three Regions for Space-Time Spectrum Sensing and Access in Cognitive Radio Networks,” IEEE Transactions on Vehicular Technology, vol. 64, no. 6, pp. 2448-2462, Jun. 2015. Article (CrossRefLink)

[28] Wikipedia of Hölder's inequality: https://en.wikipedia.org/wiki/Höl4der's_inequality 


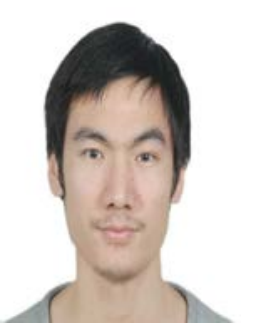

Zhiqing Wei received his B.S. and Ph.D. degrees from Beijing University of Posts and Telecommunications (BUPT) in 2010 and 2015 respectively. Now he is a lecture at BUPT. His research interests are network information theory and optimization of wireless networks such as cognitive radio networks and unmanned aerial vehicle networks.

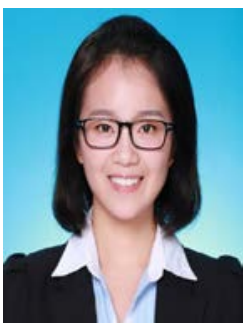

Huici Wu received her B.S. degree in Information Engineering School from Communication University of China, Beijing, China, in 2013. She is currently pursuing Ph.D. degree with the communications and information systems at Beijing University of Posts and Telecommunications, Beijing, China. From September 2016 to August 2017, she is visiting the Broadband Communications Research (BBCR) Group, Department of Electrical and Computer Engineering, University of Waterloo, Waterloo, ON, Canada. Her research interests are in the area of wireless communications and networks, with current emphasis on the cooperation and physical layer security in heterogeneous networks

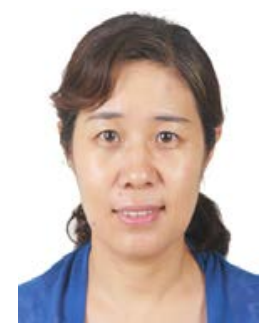

Zhiyong Feng received her B.S., M.S. and Ph.D. degrees from Beijing University of Posts and Telecommunications (BUPT), China. Now she is a professor at BUPT and she is the Director of Key Laboratory of Universal Wireless Communications, Ministry of Education, P. R. China. She is a senior member of IEEE and active in standards development such as ITU-R WP5A/WP5D, IEEE 1900, ETSI and CCSA. Her main research interests include the wireless network virtualization in 5th generation mobile networks (5G), spectrum sensing and dynamic spectrum management in cognitive wireless networks, universal signal detection and identification, network information theory, etc. 